\title{
La construcción individual del sistema de numeración convencional *
}

\section{Mercè Bassedas ** Rosa Sellarès}

Instituto Municipal de Investigación en Psicologia Aplicada a la Educación. Barcelona

La apropiación de un objeto cultural, en este caso el sistema de numeración posicional, supone un proceso de construcción por parte del individuo que presenta en algunos casos grandes similitudes con el proceso histórico de construcción. Las autoras de este artículo han emprendido una investigación que constituye un acercamiento a esos procesos y sus posibles derivaciones pedagógicas. 
El niño está en contacto con la cultura mucho antes de que la escuela se la transmita de forma organizada. El aprendizaje escolar no 'parte nunca de cero ya que siempre se ve precedido por las ideas que el niño tiene acerca de aquello que se le va a enseñar. Así sucede con las primeras nociones aritméticas: antes de acudir a la escuela, y a partir de su experiencia y su actividad sobre los objetos que le rodean, el niño ha tenido ya la oportunidad de elaborar ciertas hipótesis acerca de las cantidades y de cómo se representan.

Una de las actividades a la que el niño se entrega muy pronto y con gran entusiasmo es contar. Con ella aprende a individualizar y a ordenar los objetos, asf como a dar sentido a la serie de números que aprende a recitar precozmente en casa y en la escuela. La práctica del contaje verbal prepara el acceso a la comprensión de la numeración escrita. El niño conoce muy pronto la existencia de las cifras; pero éstas adquieren mayor sentido cuando comprende que expresan las cantidades contadas.

La numeración escrita, al igual que el lenguaje escrito, es un sistema gráfico culturalmente establecido que transcribe mediante signos el pensamiento, con la doble función de representación y de comunicación. Se trata, en uno y otro caso, de objetos culturales, indispensables para el individuo en tanto que medios de adaptación social e instrumentos para la adquisición de conocimientos, por ello, la escuela los transmite simultáneamente y lo antes posible. Entre un sistema y otro, sin embargo, existen considerables diferencias que pọr ser obvias para el adulto se considera que también deben serlo para el niño de seis años. Mientras la escritura traduce por medio de letras los fonemas de lenguaje oral, el sistema de numeración es ideográfico, representa una idea y prescinde de su expresión verbal. La estructura interna de ambos sistemas es distinta: la combinatoria determinada por el valor posicional de las cifras en el sistema de numeración es más compleja que la del lenguaje escrito, en el que las letras siempre tienen el mismo valor cuantitativo.
La comprensión de las propiedades de los sistemas gráficos convencionales es imprescindible para su correcta utilización. En el caso del sistema numérico ésta supone no sólo la aplicación mecánica del sistema sino también la posibilidad de comprender los algoritmos de las operaciones elementales y de manejar las diferentes bases de numeración.

Piaget y su escuela enfocan la cuestión de la adquisición de conocimientos desde una perspectiva constructivista: la construcción de un conocimiento -tanto a nivel ontogenético como filogenético- supone un proceso; no es posible el paso inmediato de la ignorancia al saber. La historia de la numeración nos muestra que antes de la invención del sistema posicional de origen indio adoptado universalmente en nuestros días, la humanidad utilizó otros sistemas de menor complejidad. Estos tomaron distintas formas según el desarrollo técnico-cultural y las circunstancias histórico-sociales de los pueblos que los creaban. En el origen de todos ellos estuvo, sin embargo, la necesidad de tener un registro del contaje verbal.,

La apropiación individual de un objeto cultural como el sistema de numeración supone. también un proceso. El niño, como cualquier sujeto que aprende, no se limita a almacenar conocimientos, sino que los construye activamente. Esta construcción la realizará partiendo tanto de lo que se le enseñe como de lo que él descubra acerca de este sistema gráfico convencional, dado que forma parte del mundo que le rodea, y como todo elemento de su entorno, le interesa. De esta forma, el proceso de apropiación individual del sistema numérico convierte el objeto cultural en objeto de conocimiento a asimilar de forma progresiva por las estructuras intelectuales infantiles.

El estudio cuyas principales conclusiones describimos a continuación, surgió de nuestro propósito de estudiar las distintas etapas de dicho proceso de reconstrucción. A partir del análisis de producciones gráficas espontáneas, nos propusimos establecer una génesis. 
Ơtra cuestión que nos interesaba era estudiar las posibles semejanzas entre algunos aspectos de las producciones infantiles y los sistemas de numeración que se han sucedido históricamente.

\section{METODOLOGIA}

\section{Población}

La muestra, formada por 100 niños $-50 \mathrm{ni}$ ños y 50 niñas - de edades comprendidas entre 6 y 10 años ( \pm 3 meses), se extrajo de una población de niños barcelóneses de clase social media, que recibían una enseñanza activa. Los sujetos se eligieron en función de su fecha de nacimiento, y se tomaron 20 niños por edad.

\section{Situación experimental}

La situación experimental consistía en una entrevista clínica realizada de forma individual y dentro del marco escolar. El núcleo central de ésta consistía en pedir al niño que inventara una forma de anotar en el papel lo que contaba, que fuera muy diferente a la que ellos conocían. Finalizada la tarea se le pedra que aplicara el usistema» inventado a algunas cantidades que se le dictaban.

\section{Método de análisis de los resultados}

$\mathrm{El}$ análisis de las producciones gráficas obtenidas se emprendió teniendo en cuenta los siguientes aspectos:

Ṕroximidad del «sistema» propuesto por el niño al sistema de numeración convencional. Dicha proximidad se valora en el sentido de si en su producción el niño contempla la posibilidad de combinar los grafismos o no, y en caso afirmativo, si las leyes de combinación son tan sólo de tipo aditivo o si incluyen el valor posicional.

Número de grafismos originales empleados para representar las cantidades.

Estabilidad de los grafismos, en el sentido de si se respeta el valor otorgado a cada grafismo a lo largo de.toda la producción.

Características formales de los grafismos empleados.

Posibilidad de aplicación a las diferentes cantidades de los grafismos propuestos en un primer momento.

Inclusión o no del cero entre dichos grafismos $y$, en caso afirmativo, momento de la inclusión.

\section{ANALISIS DE LOS RESULTADOS}

La codificación de las producciones obtenidas en base a los criterios arriba expuestos ha dado lugar a la siguiente clasificación de las conductas (véase cualdro I):

Cuadro: I

Distribución de las conductas descritas en $\%$

\begin{tabular}{|c|c|c|c|c|c|c|c|}
\hline Conductas & 1 & 2 & 3 & 4.1 & 4.2 & 5 & 6 \\
\hline 6 & 55 & 15 & & 30 & & & \\
\hline 7 & & 20 & & 70 & & 10 & \\
\hline 8 & & & 15 & 25 & 5 & 10 & 45 \\
\hline 9 & & & 15 & 25 & 5 & 25 & 30 \\
\hline 10 & & & 5 & 10 & 10 & 30 & 45 \\
\hline
\end{tabular}




\section{Conductas sin combinatoria ni esta- bilidad}

Las conductas de este apartado, aparecen a sólo los seis años y como conducta predominante. Ponen de manifiesto la frecuentación del sistema de numeración universal por parte del niño asI como algunas de las hipótesis que éste ha podido construir acerca de él. Consisten en hacer algunos grafismos originales o tomados de otros sistemas gráficos para representar algunas cantidades aisladas y en seguir inventando tantos nuevos grafismos como cantidades se deban transcribir sin considerar en ningún momento la posibilidad de que las cantidades pueden ser representadas por medio de un limitado conjunto de cifras. Un niño justifica su producción en estos términos: «Hago cada vez un número nuevo porque ningún número es igualm. La producción de Ja $(6 ; 0)$ que utiliza letras del alfabeto, ilustra este tipo de conductas.

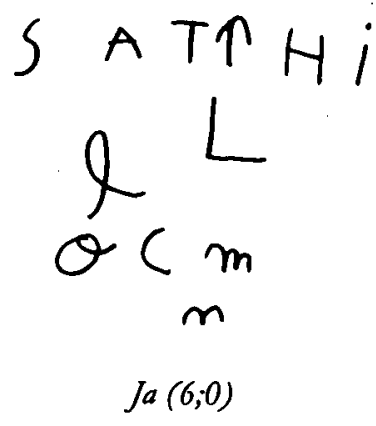

En algunos casos -y de forma concordante con el pensamiento sincrético propio de esta edad- el niño supone que entre la cantidad y el grafismo que la representa debe existir una relación directa, de tipo intensivo, que le lleva a adecuar el tamaño de su «cifra» a la cantidad que representa. Ảsí, al pedir a uno de los niños -Ma $(6 ; 1)$ - que anote a su manera el número treinta, después de haber representado otros de menor valor numérico, dice: «Este tendrá que ser más grande» y actúa consecuentemente a su comentario.
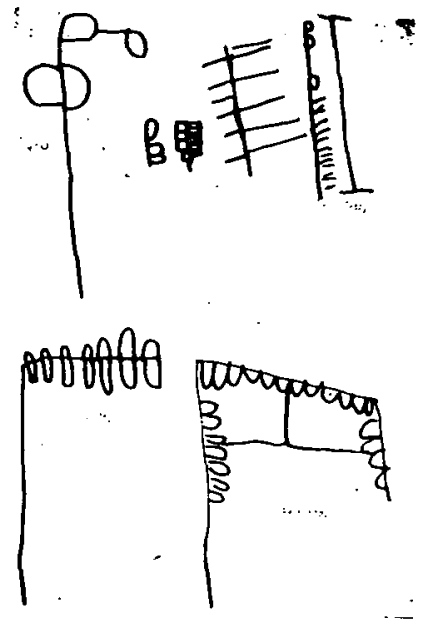

$M a(6 ; 1)$

\section{Conductas con cifras «árabes»}

Estas producciones que aparecen tan sólo a los seis y siete años consisten en introducir variaciones mínimas en el sistema de notación universal de las cifras. La dificultad de desligarse del sistema gráfico convencional lleva a modificar el grafismo convencional de las cifras "camuflándolas" o inviertiendo su orientación como hace $\operatorname{Dav}(7 ; 2)$.

\section{$\varepsilon-Z-7-9-P+A \varepsilon$ $\operatorname{Dav}(7 ; 2)$}

En algún caso se propone cambiar el valor asignado a las cifras aunque dicho cambio no se mantenga luego en el momento de combinar los signos, como en el caso de Ber $(6 ; 3)$.

\section{9}

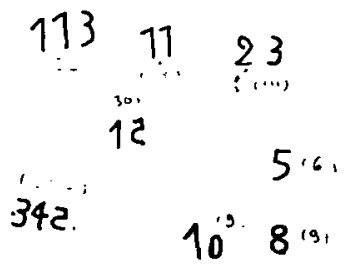

$\operatorname{Ber}(6 ; 3)$ 


\section{Conductas sin combinatoria y con estabilidad}

Esta conducta que aparece a los ocho, nueve y diez años se caracteriza por inventar un signo diferente para cada número sin contemplar su combinatoria. Este hecho recuerda las realizaciones de los niños de seis años. La diferencia entre las producciones de ambos grupos de edad reside, sin embargo, en que mientras las de los más jóvenes carecen de estabilidad, las que comentamos gozan de ella ya que el grafismo inventado en un primer momento se mantiene a lo largo de toda la producción. Algunos niños se inclinan por utilizar algunas de las letras que traducen la expresión verbal del número, otros emplean un recurso especial como hace Je $(9 ; 1)$ que rcurre a un signo más o menos complicado y grande según el número a representar:

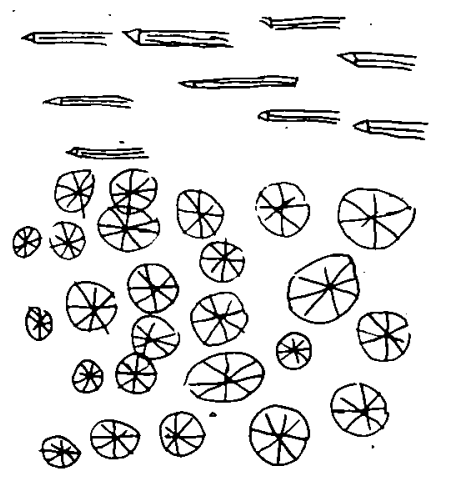

$$
J e(9 ; 1)
$$

Otros acuden el dibujo figurativo y utilizan un dibujo distinto para cada cantidad. Frecuentemente se establece una relación simbólica entre el dibujo y el número que éste representa, Mar (10;0), por ejemplo, dibuja una manifestación para transcribir el númro mil.

\section{Conductas aditivas}

En las producciones incluídas en este apartado las cantidades se componen mediante la

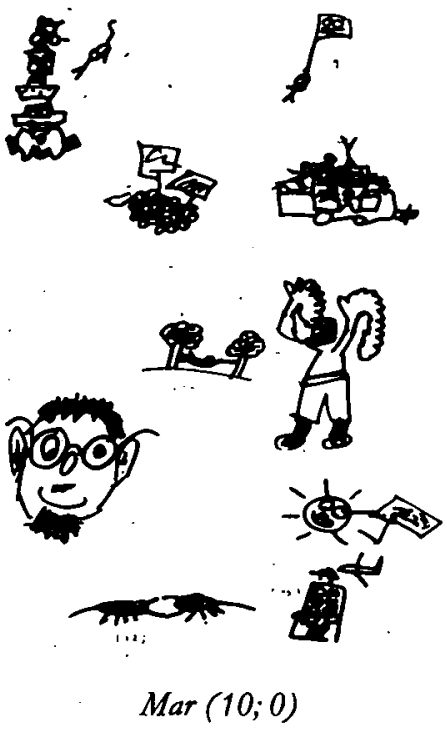

suma de elementos sin que se contemple aún la posibilidad de que un signo adopte un valor $\mathbf{u}$ otro según la posición que ocupe. Entre las conductas aditivas se incluyen tanto las producciones de los sujetos que se sirven de la correspondencia, como las de los que recurren a la invención de grafismos de distintos valores que yuxtaponen hasta alcanzar la cantidad deseada.

\subsection{Conductas de correspondencia}

Esta conducta predomina a los siete años, cuando la seguridad en el manejo y comprensión del esquema de correspondencia -que permite asegurar la equivalencia de dos conjuntos puestos previamente en correspondencia, a pesar de las transformaciones figurales que pueden afectar a ambos conjuntos- determina la consolidación de la noción de cantidad en el niño.

Históricamente, el principio de correspondencia está en la base de las formas más primitivas de registro de la cantidad. Los sumerios; por ejemplo, se servían de él como instrumento para el recuerdo del contaje. Por cada cabeza de ganado depositaban una bolita de arcilla en un recipiente y añadían una o sacaban otra cuando por alguna razón variaba 
la cantidad global. Este sistema les permitfa saber en todo momento de cuántas reses disponían.

La producción de $\operatorname{Em}(7 ; 0)$, que hace tantos dibujos como unidades contándolos a continuación para asegurarse de que ha alcanzado la cantidad pedida, ilustra este tipo de conducta tan generalizada a esta edad.

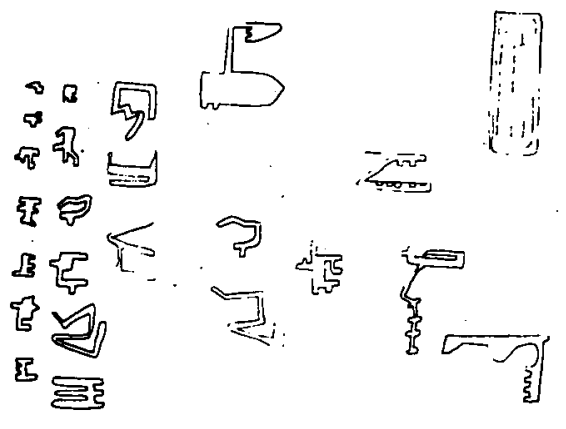

$\operatorname{Em}(7 ; 0)$

Otros niños utilizan grafismos esquemáticos cualesquiera (rayas, puntos...), como ilustra la producción de Dan $(7 ; 7)$, para representar las cantidades pedidas.

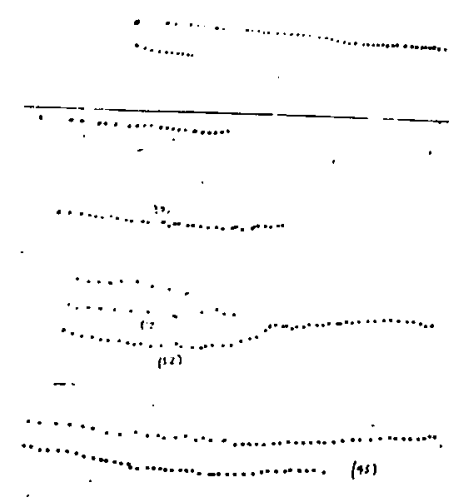

$\operatorname{Dan}(7 ; 7)$

Los niños algo mayores que recurren a la correspondencia al iniciar la tarea son capaces de modificar sus producciones en el transcurso de ella. Para conseguir un ahorro en el número de grafismos a realizar, introducen otros que representan grupos de unidades. Esto hace por ejemplo Dav $(8 ; 11)$ quien para representar 1.450 utiliza: $X$ para 1000, + para $100 \mathrm{y}$ | para representar las unidades.

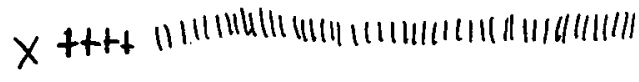

Dav $(8 ; 11)$

\subsection{Conductas que preveen un código con signos especiales para las decenas.}

Estas conductas, que aparecen a los ocho años, son aditivas como las de correspondencia, pero se diferencian de éstas porque desde el inicio preveen un código que además de los grafismos que representan las unidades incluye signos especiales paraladecena y sus potencias. Las propuestas de representación recurren a una estrategia muy parecida ạ la utilizada por los egipcios en el sistema de numeración jeroglf́fico de base diez en el cual, y según el principio aditivo, cada símbolo se repetía tantas veces como fuera necesario; el número 12.105 por ejemplo se transcribía de la forma siguiente:

\section{105. $=78 \&$ \&}

X $a v(7 ; 10)$ representa las cantidades de modo similar yuxtaponiendo cuadrados de distinto tamaño y barras; el número 95 , por ejemplo, se compone de $1 \square$ que representa 50 , de $4 \square$ cada uno de los cuales simboliza una decena y de 5 barras:

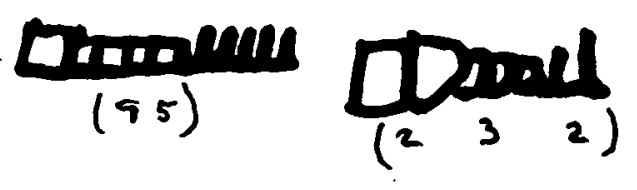

$X a v(7 ; 10)$ 
5. Conductas que intentan la transposición del sistema de numeración universal .

Las conductas que integran este apartado aparecen a partir de los siete años y se caracterizan por el hecho de que intentan reproducir las leyes de combinatoria de los signos del sistema de numeración universal con grafismos distintos a las cifras convencionales. Ciertas limitaciones hacen, sin embargo, que dicho intento se vea frustrado.

Una de las limitaciones que aparece es la persistencia de aspectos aditivos junto a la utilización del valor posicional. Es frecuente encontrar producciones en las que, junto a la utilización de los dígitos y del 0 , se introducen signos especiales para la decena y sus potencias; Jor (9; 9), por ejemplo, representa 12.103 del siguiente modo:

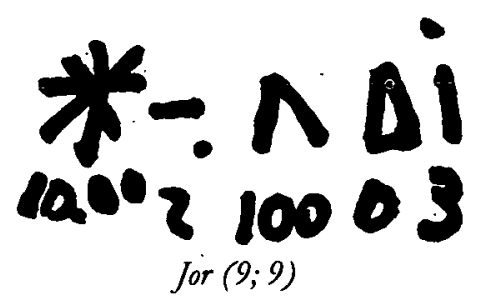

La presencia de lo aditivo se observa también en el caso de los sujetos que empiezan su producción utilizando una estrategia aditiva pero acaban recurriendo a una transposición imperfecta, o entre aquellos que alternan o simultanean la conducta aditiva y la posicional durante toda la tarea, de las que Jo $(10 ; 1)$ es un ejemplo:

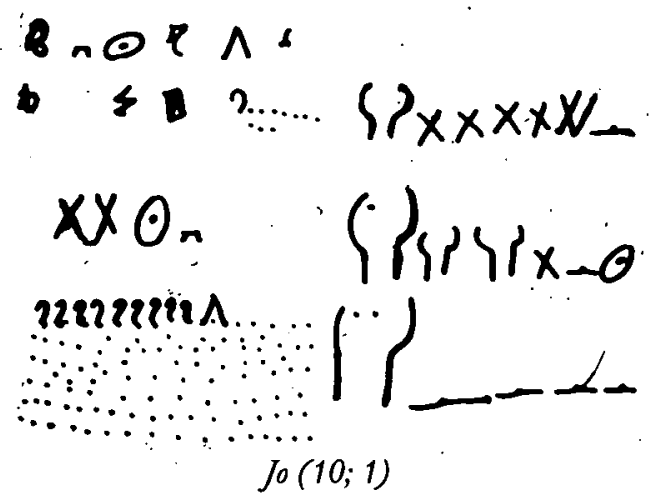

Otra limitación que presentan las conductas de este tipo consiste en la dificultad de incluir el 0 . A menudo no se prevee un grafismo que lo represente en el momento de pensar en el código. En algún caso se introduce al final de la producción ante la necesidad de transcribir cantidades muy altas. Un ejemplo de intento de utilización del valor posicional sin incluir el 0 lo encontramos en Anb (9; 2):

\section{.XIV V-XIII II. (1450) (12.103)}

Anb $(9 ; 2)$

La no inclusión o la inclusión tardía del 0 en las producciones infantiles recuerda las dificultades que la humanidad ha experimentado repetidamente a lo largo de la historia para considerar el 0 como un número más y para introducirlo en los sistemas de numeración posicional que incluía el 0 medial para marcar la ausencia de una de las potencias de la base en el interior de un número. Desconocían, en cambio, el papel del 0 terminal como operador que multiplica el valor de las cifras que le preceden por la base de la numeración: para representar sesenta, base de su numeración, utilizaban el mismo empleado para la unidad pero aumentando su tamaño.

\section{Conductas de transposición del sis- tema de numeración universal}

Este apartado reúne las producciones que -como las de $\mathrm{Ma}(10 ; 1)$ o Sol $(10 ; 2)-$ consisten en elaborar un código, paralelo al de las cifras árabes, compuesto por diez signos diferentes que representan el $0 \mathrm{y}$ los dígitos y aplicarlo correctamente para la representación de las cantidades pedidas. Esta transposición, que tiene en cuenta todas las leyes del sistema de numeración posicional uitilizado universalmente, aparece ya a los ocho años, pero no llega a ser mayoritaria hasta los diez años, edad en que ya es posible comprender y 
utilizar las leyes del sistema de numeración decimal.
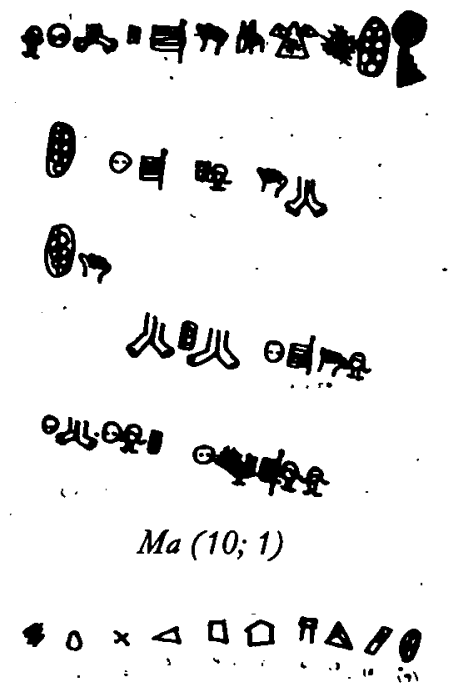

a. 5

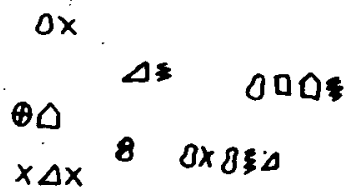

$08 \Delta 0 \$ 33$

Sol $(10 ; 2)$

De la presencia preponderante de las conductas de transposición, no creemos, sin embargo, que se pueda deducir que todos los sujetos de esta edad sean capaces de inventar ex-nihilo un sistema de la complejidad del sistema de numeración posicional, ya que reproducir no es lo mismo que inventar. Lo que muestran estas conductas que comentábamos es que el träbajo conceptual individual sobre el sistema de numeración posicional permite ya su comprensión y generalización.

\section{CONCLUSIONES}

En el momento de iniciar el trabajo cuyos resultados generales acabamos de describir partramos de la idea de que el niño, al iniciar la escolaridad se ve forzado.a utilizar un obje- to cultural, el sistema de numeración posicional, que por sus características implica unas operaciones matemáticas que desbordan sus posibilidades intelectuales. De acuerdo con la teoría de Piaget, pensábamos que forzosamente debía haber un proceso de reconstrucción individual del sistema de numeración, que permitiera al niño llegar a la comprensión real de éste.

Este proceso es el que hemos podido estudiar a través de las producciones espontáneas con las que los niños respondían a nuestra consigna. Efectivamente, hemos comprobado que las producciones gráficas de los niños muestran la existencia de una génesis en la comprensión del sistema de numeración convencional. Las sucesivas conductas nos ayudan a comprender este proceso de construcción individual, y a través de ellas vemos como el sistema de numeración impuesto por la cultura se convierte progresivamente y a través del constante diálogo entre el individuo y el medio, en una pertenencia del sujeto.

Si consideramos -también siguiendo a Piaget- que la comprensión de un objeto de conocimiento comporta la posibilidad de reconstruirlo, podremos decir que el niño ha comprendido el objeto de conocimiento «sistema de numeración posicional de base 10 ", en el momento en que pueda reconstruir los elementos que lo constituyen, y las leyes de composición de estos elementos en una situación que requiera la utilización del sistema.

Esta posibilidad de reproducir el sistema de numeración significa tener en cuenta la relación entre las cifras y las cantidades que éstas representan, así como las operaciones aritméticas implícitas en el sistema. Ello supone:

a) La previa estructuración de la sucesión de los números enteros positivos de acuerdo con la relación iterativa que los une y que hace que cada número incluya a los que le preceden. El valor inclusivo concedido al número y al grafismo que lo representa es lo que permite la utilización limitada de grafismo para la re- 
presentación de las cantidades que está en la base de la invención de todo sistema de numeración. Por el contrario el registro de la cantidad basado en el principio de la correspondencia, que serfa la forma más primitiva de notación de cantidades, no contempla el valor inclusivo de los signos, ya que cada grafismo corresponde a un elemento de los que forman la cantidad total.

b) La presencia de la idea de código, es decir, de un sistema de obligaciones que comporta el conocimiento de unos signos, los cuales gozan de estabilidad y se combinan de acuerdo con unas reglas.

c) La comprensión de la coexistencia de las operaciones de adición y de multiplicación en las reglas que rigen la combinación de los dígitos. Efectivamente, el sistema de numeración que empleamos habitualmente tiene como finalidad la representación de todos y cada uno de los números enteros positivos, resultantes de la suma de unidades, decenas y centenas, etc., multiplicadas por coeficientes cuyos valores van del 0 al 9 (por ejemplo, el número 245 significa $[2 \times 100]+[4 \times 10]+[5 \times 1])$.

El principio posicional hace que sólo sean representados los coeficientes del polinomio decreciente que expresa el número y que el valor de cada cifra dependa del lugar que ocupe.
La elaboración de cada uno de estos aspectos debe darse en el niño de forma progresiva. La presión de lo cultural está presente en igual forma a lo largo de todo el proceso de construcción de este objeto de conocimiento que es el sistema de numeración. Sin embargo, y según parece desprenderse de los resultados que hemos obtenido a partir del análisis de las conductas espontáneas de los niños, unos aspectos son más fáciles que otros, por lo que su integración y su coordinación se hace de forma progresiva y en función del nivel alcanzado por las estructuras intelectuales del sujeto.

Si se analizan las producciones halladas y la génesis por ellas descrita, se observa que es posible agruparlas en tres grandes tipos a los que hemos denominado respectivamente, de aproximación formal al sistema (a), aditivas (b) y de transposición (c), que se correspondan con la progresiva estructuración y toma de conciencia de los diferentes aspectos del sistema numérico posicional. (Véase cuadros I y II.)

\section{a) Conductas de aproximación formal al sistema}

En este apartado incluiríamos las conductas sin combinatoria ni estabilidad (1), las conductas con cifras (2) y las conductas sin combinatoria y con estabilidad (3). Lo característico de estas conductas que constituyen el $25 \%$ del total de la muestra es el retener del sistema numérico convencional tan sólo el as-

\section{Cuadro II}

Distribución de los tres grandes tipos de conductas en $\%$

\begin{tabular}{|c|c|c|c|}
\hline Edad Conductas & a) Aproximación & b) Aditivas & c) Transposición \\
\hline \hline 6 & 70 & 30 & 0 \\
7 & 20 & 70 & 10 \\
8 & 15 & 30 & 55 \\
9 & 15 & 30 & 55 \\
10 & 5 & 20 & 75 \\
\hline TOTAL & 25 & 36 & 39 \\
\hline
\end{tabular}


pecto formal consistente en atribuir uno o dos grafismos distintos a cada número, sin posibilidad de combinar dichos grafismos.

Este tipo de conductas decrece con la edad: son muy numerosas $(70 \%)$ a los seis años y casi inexistentes $(5 \%)$ a los diez años. También en función de la edad adoptan una forma $u$ otra. A los seis años el intento de acomodación al sistema notacional frecuentado si bien no comprendido, lleva a la mayoría de los sujetos a no preveer ni la combinatoria ni la estabilidad de los grafismos. Esta conducta desaparece a partir de los siete años, edad en que las conductas de los niños agrupadas en este apartado $(20 \%)$ se caracterizan por la imposibilidad de desligarse del sistema convencional aprendido en la escuela y de abstraer de él sus propiedades fundamentales para su generalización.

A partir de los ocho años las producciones pertenecientes a este grupo toman del sistema numérico convencional la estabilidad de los signos, aunque continúan sin combinar grafismos para representar las cantidades. Lo que proponen es más una enumeración que un sistema, ya que a cada número le hacen corresponder un grafismo diferente.

\section{b) Conductas aditivas}

Este apartado agrupa las conductas de correspondencia (4.1) y las de código con signos especiales para las decenas (4.2), las cuales se sirven de estrategias aditivas para la representación de cantidades. Este tipo de conductas tiene un máximo de frecuencia a los siete años $(70 \%)$ y están presentes, si bien en forma decreciénte, en todas las edades, constituyendo el $36 \%$ del total de las producciones.

En todos los niños de seis y siete años las conductas aditivas adoptan la forma de la correspondencia. Antes de pensar en un «sistema» de numeración en el que estén previstos el valor inclusivo $y_{2}$ la posibilidad de combinación de los grafismos, el niño recurre al principio de correspondencia que es el que utiliza espontáneamente $y$ a nivel práctico para manejar las cantidades.

Esta conducta constituiría, tal como hemos dicho anteriorn tte la forma más primitiva de registro de la cantidad. Más adelante, a partir de los ocho años estas conductas de correspondencia evolucionan hacia sistemas aditivos estructurados con los que coexisten.

La mayoría de las civilizaciones que nos han precedido han recurrido, como estos sujetos cuyas producciones recuerdan los sistemas egipcio o azteca, a principios aditivos para la construcción de sus sistemas de notación de cantidades. Cabe destacar el hecho de que de veinticuatro sistemas de numeración originales atestados, tan sólo cuatro de ellos (Maya, Babilónico, Indio [Gwailor] y Chino), recurren al principio del valor posicional.

\section{c) Conductas de transposición}

Como síntesis del trabajo de construcción intelectual individual y de la acomodación al sistema cultural, hallamos este tercer grupo de conductas ( 5 y 6 ) que constituyen el $39 \%$ de la muestra y se caracterizan por la posibilidad de generalizar las leyes propias del sistema de numeración posicional. A los ocho y nueve años son un $55 \%$ los sujetos que recurren a estas conductas, aumentando hasta un $75 \%$ a los diez años. A partir de los ochonueve años el desarrollo intelectual alcanzado por los niños permite ya manejar con cierta seguridad la sucesión de los números naturales y pensar en sistemas, es decir, en conjuntos de reglas para representarlos. Los contenidos escolares exigidos a estas edades también contribuyen a impulsar la apropiación del sistema de numeración convencional.

A pesar de la evolución intelectual y de la presión escolar, la transposición del sistema de numeración posicional con todas sus características no se consigue de forma inmediata. Si bien a partir de los ocho años son máyoria los sujetos que la intentan, también son muchos los que no la consiguen. Las oscilaciones que éstos presentan nos hacen pensar en las dificultades que nuestro sistema plantea al escolar. En las conductas de inicio de combinatoria (5), previas a las de transposición (6), se evidencia una persistencia de aspectos aditivos que lleva por ejemplo, a no incluir el 0 en el sistema o a preveef un signo para represen- 
tar la decena al mismo tiempo que se utiliza el 0 .

Las dificultades de los niños son comparables a las que tuvieron que vencer algunos pueblos en su avance hacia la numeración de posición. Un ejemplo lo tenemos en la India (numeración de Gwailor) donde la creación del sistema que hoy utilizamos habitualmente y cuya existencia está atestada desde el siglo IX después de J. C. fue posible gracias a la evolución sufrida en un sistema aditivo que renunció a los signos originales para representar las distintas potencias de la base.

A través de las distintas conductas descritas se observa tanto el continuo trabajo conceptual individual como la progresiva integración de los aspectos que presenta el sistema de numeración posicional.

En un primer momento, representado por las conductas más elementales, que hemos denominado de aproximación al sistema, el niño sólo toma de éste la idea de que ciertos grafismos representan cantidades. A partir de cierta edad el niño considera necesario otorgar estabilidad a dichos grafismos.

Las conductas aditivas que en un segundo momento sustituyen a las de aproximación, reflejan de forma especial las posibilidades intelectuales de los sujetos que las proponen. Este tipo de conductas parece testimoniar que, a pesar del modelo cultural que le es enseñado, el individuo recurre a formas de notación más simples y próximas a sus estructuras operatorias, lo que da lugar a la utilización masiva de la correspondencia a los siete años. Más adelante estas conductas evolucionan y preveen la utilización de una cierta cantidad de grafismos que goza de estabilidad y que deben ser combinados de una forma determinada -es decir, de un sistema-. Las reglas de combinación de dichos grafismos, sin embargo, se basan únicamente en la adición.

Finalmente, los niños son capaces de generalizar el sistema de numeración posicional con todas sus características, es decir, la estabilidad de los signos y la organización de estos en un sistema que contempla tanto los as- pectos aditivos como los multiplicativos. Hemos visto, sin embargo, que la posibilidad de transposición no aparece de forma espontánea sino que se ve precedida por intentos que muestran las dificultades del niño para la comprensión del sistema de numeración posicional.

En cuanto a la cuestión planteada en la introducción sobre la similitud entre el proceso de adquisición del sistema de numeración por parte del niño y los procesos de construcción histórica de los sistemas de numeración, observamos que tanto en el niño como en la evolución histórica existe un proceso de construcción en el que el progreso es la ley. $\mathrm{El}$ individuo, al igual que las culturas, avanza paulatinamente hacia la mejora de sus instrumentos intelectuales.

La comparación entre ambos procesos debe ser prudente y de ningún modo se puede esperar encontrar una recapitulación de los distintos sistemas de numeración históricos en la génesis individual. Existe una diferencia fundamental entre el proceso de invención cultural y el de reinvención individual, que consiste en la distinta motivación que guía uno y otro. Mientras que el primero está motivado por unas determinadas necesidades históricas y científicas -los sistemas de numeración posicionales se crearon en círculos intelectuales en los que los sabios buscaban recursos para transcribir y recordar los grandes números que exigían sus cálculos astronómicos-, el segundo lo está por la necesidad del individuo infantil de apropiarse de los instrumentos que la cultura le ofrece para una mejor adaptación. La comprensión real de estos instrumentos, de los que el sistema de numeración sería un ejemplo, no exige que el individuo los invente ex-nihilo, pero sf que sea capaz de encontrar por sí mismo las razones y leyes implícitas por las que ellos se rigen, y por tanto de reinventarlos por lo menos en parte.

A pesar de las diferencias entre el proceso de invención histórica de sistemas de numeración y el de la construcción individual del 
Sistema de numeración posicional se dan una serie de hechos generales coincidentes. Una primera coincidencia que se constata es el predominio de estrategias aditivas para representar la cantidad. El peso de los sistemas aditivos en la historia -doce de las veinticuatro numeraciones originales atestadas son de tipo aditivo- y la signiticativa frecuencia de estas conductas en los sujetos entrevistados ( $36 \%$ ) parece dar constancia de la similitud entre los procesos intelectuales de algunos pueblos en su desarrollo histórico y los de los sujetos de la operatividad concreta.

Otro hecho coincidente sería la similitud de los titubeos que preceden a la aparición de los sistemas posicionales. La creación histórica de éstos supuso vencer dificultades como el abandono de la representación original de las potencias de la base o la integración del cero en el sistema, que son las mismas que encontramos en los sujetos que intentan la transposición.

Si, como puede inferirse de los resultados encontrados, la comprensión del sistema de numeración posicional supone una construcción individual, nos podríamos preguntar cuál es el papel que el aprendizaje escolar tiene en todo el proceso de construcción de conocimientos y en el del-sistema de numeración en particular.

Una posibilidad, a la que se atiene la escuela tradicional, es considerar que el sistema de numeración se debe transmitir lo antes posible y que el niño debe ser capaz, también lo antes posible, de utilizarlo aunque sea de forma mecánica. Otra posibilidad serfa aplazar cualquier intervención pedagógica hasta que la capacidad intelectual del sujeto le permita la comprensión adecuada. En el caso del sistema de numeración posicional, y según nuestros datos, ello supondría retrasar el aprendizaje de la representación gráfica convencional de la serie numérica. Una tercera posibilidad, por la que abogariamos, consiste en hacer que los aprendizajes respeten, estimulen y no inhiban la génesis espontánea de cualquier conocimiento con la finalidad de que el niño utilice con la máxima comprensión aquello que se le enseña. Según este enfoque, la escuela no retrasa el aprendizaje sino que conoce y sigue de cerca la construcción individual del conocimiento y propone situaciones que logran la elaboración intelectual espontánea por parte del niño.

En el caso de la enseñanza del sistema de numeración posicional habría que tener en cuenta, antes de que el individuo pueda servirse de él como soporte de la conceptualización de cantidades, -diff́cilmente podriamos. representarnos cantidades importantes sin la ayuda de su representación escrita-, debe superar un complejo aprendizaje en el que las dificultades propias de la construcción conceptual de la serie numérica interfieren con las que comporta la comprensión de su representación gráfica convencional.

El respeto por la construcción individual del sistema de numeración implica no imponerlo directamente, sino estimular al niño para que trabaje sobre todos aquellos aspectos que favorecen la comprensión de la numeración. La consolidación a nivel de acción de aspectos previos como la correspondencia, o implícitos en el sistema de numeración, como los aspectos multiplicativos, permitirá al niño pasar a un plano de abstracción superior como el que supone la representación gráfica. Un principio general, válido también aquf, es que la acción debe preceder a la formalización. Cuanto más actúe el niño de forma inteligente sobre la realidad, más posbilidades tendrá de reflexionar y reorganizar en un plano superior lo que ha conseguido en el de la acción. No provocar prematuramente la formalización recurriendo a un sistema tan complejo como nuestro posicional sino respetar la representación gráfica espontánea, permite que los niños descubran la necesidad y utilidad de la representación de la cantidad y estimula la confrontación y discusión de las diferentes hipótesis que aquellos tengan sobre el sistema de numeración convencional, haciéndolo, así, comprensible. 


\section{Bibliografia consultada}

Colette, J. P. Histoire des matbématiques. Ottawa: Editions du Rénovation Pédagogique Inc. Ottawa, 1979.

Ferreiro, E.; Teberosky, A. Los sistemas de escriture en el desarrollo del niño. Méjico: Siglo XXI, 1979.

Gómez, C. Procesos cognitivos en el aprendizaje de la multiplicación, Madrid: Infancia y Aprendizaje, 1981, 15.

Gurrel, G. Histoire comparée des numérations écrites. Paris: Flamarion, 1975.

Meljac, C. Décrire, agir, compter. Paris: P.U.F., 1979.

Moreno, M.; SASTRE, G. Aprendizaje y desarrollo intelectual. Barcelona: Gedisa, 1980.

Morf, A. Recherches sur l'origine de la connexité de la suite des premiers nombres. Paris: Etudes d'Epistemologie Génétique n.o XIII, P.U.F., 1962.

Piager, J. (1950). Introducción a la Epistemologia Genética. El pensamiento matemático. Buenos Aires: Paidos, 1975.

Piaget, J.; Szeminska, A. La genèse du nombre chez l'enfant. Neuchâtel: Delachaux et Niestlé, 1959.

Pinget, J.; Inhelder, B. L'image mentale chez l'enfant. París: P.U.F., 1966.

PInGet, J. (197.5). La equilibración de las estructuras cognitivas. Problema central del desarrollo. Madrid: Siglo XXI, 1978.

Pinget, J. y otros. La enseñanza de las matemáticas modernas. Madrid: Alianza Universidad, 1978.

Sastre, G.; Bassedas, M.; Sellares, R. La teoria de Piaget y la investigación psicopedagógica. En: La Psicologia. genética de Jean Piaget. Barcelona: Anuario de Psicologfa de la Universidad de Barcelona, 1981, 223-245.

SASTRE, G.; Moreno, M. Descubrimiento y construcción de conocimiento. Barcelona: Gedisa, 1980.

SChmandt-Besserat, D. El primer antecedente de la escritura. Investigación y Ciencia, 1978, 31.

Vergnaud, G. L'enfant, la mathématique et la réalité. Berna: Peter Lang, 1981.

\section{Resumen}

El sistema de numeración posicional de base 10 constituye un objeto cultural, resultado de un largo y dificultoso desarrollo bistórico, que se convierte en un objeto de conocimiento a asimilar por el niño. En este artículo se estudia el proceso de reconstrucción individual de este instrumento intelectual, a través de las producciones gráficas espontáneas de 100 niños de edades comprendidas entre los 6 y los 10 años, obtenidas en situación de invención de sistemas de notación de cantidades. Las producciones de los niños, cuyas estrategias recuerdan en mucbos casos las empleadas en los sistemas de numeración bistóricos, describen una génesis. Esta se extiende desde producciones que no contemplan la ostabilidad de los grafismos ni la posibilidad de combinarlos, basta las que recurren al principio del valor posicional, pasando por producciones de tipo aditivo y producciones que intentan, sin conseguirla, la transposición del sistema de numeración convencional.

\section{Résumé}

Le système de numération de base dix est un objet culturel résultat d'un long et difficulteux dévéloppement bistorique, Lequel devient un objet de connaissance à assimiler par l'enfant.

Dans cet article on étudie be processus de reconstruction individuel de cet instrument intellectuel à travers des productions grapbiques spontanées de 100 enfants de six a dix ans obtenues dans une situation d'invention de systèmes de notation de quantités.

Les productions des enfants, dont les strategies rappelent dans plusieurs occasions celles employées dans les systèmes de numeration bistoriques, décrivent une genèse. Celle ci s'étend dès praductions qui ne contemplent ni ia stabilité des graphismes ni la possibilité de les combiner jusqu'à celles qui recourrent au principe du valeur positionnel, passant par productions de type additif et productions qui présentent une combinatoire dans laquelle on essaie sans succes la transposition du système de numeration conventionnel. 


\section{Summary}

The system of position numeration in base ten is a cultural object, resulted from a long and troublesome bistoric development, which becomes an object of knowledge to be assimilated by the child.

In this paper, the process of individual reconstruction of this intelectual tool is studied through the spontaneous grafic productions on 100 children aged from six to ten obtained in a situation of invention of systems of quantity notation.

The cbildren's productions, whose strategies often remain us of those strategies used in the historic numeration systems, describe a genesis. This genesis extends from productions which do not involve the stability of the grafics nor their combination possibilities, to those which resort to the principle of the position value going through productions of additive type and productions which present a combination system in which the transposition of the conventional numeration system is attempted without success. 